\title{
FOURIER PROBLEM FOR WEAKLY NONLINEAR EVOLUTION INCLUSIONS WITH FUNCTIONALS
}

\author{
Mykola M. Bokalo, Iryna V. Skira ${ }^{\dagger}$
}

\begin{abstract}
The Fourier problem or, in other words, the problem without initial conditions for evolution equations and inclusions arise in modeling different nonstationary processes in nature, that started a long time ago and initial conditions do not affect on them in the actual time moment. Thus, we can assume that the initial time is $-\infty$, while 0 is the final time, and initial conditions can be replaced with the behaviour of the solution as time variable turns to $-\infty$. The Fourier problem for evolution variational inequalities (inclusions) with functionals is considered in this paper. The conditions for existence and uniqueness of weak solutions of the problem are set. Also the estimates of weak solutions are obtained.
\end{abstract}

Key words: Fourier problem, problem without initial condition, evolution inclusion, subdifferential of functional.

2010 Mathematics Subject Classification: 26D10, 47J20, 47J22, 49J40.

Communicated by Prof. P. I. Kogut

\section{Introduction}

In this paper we consider problem without initial conditions, or, in other words, the Fourier problem for evolution variational inequalities (inclusions) with functionals. Let us introduce an example of the problem being studied here.

Let $\Omega$ be a bounded domain in $\mathbb{R}^{n}(n \in \mathbb{N}), \partial \Omega$ be the boundary of $\Omega$, which is piecewise surface. We put $Q:=\Omega \times(-\infty, 0], \Sigma:=\partial \Omega \times(-\infty, 0], \Omega_{t}:=$ $\Omega \times\{t\} \forall t \in \mathbb{R}$. For an arbitrary measurable set $F \subset \mathbb{R}^{k}$, where $k=n$ or $k=n+1$, let $L^{2}(F)$ be the standard Lebesgue space. Let $L_{\text {loc }}^{2}(\bar{Q})$ be the space of functions defined on $Q$ such that their restrictions on any bounded measurable set $Q^{\prime} \subset Q$ belong to $L^{2}\left(Q^{\prime}\right)$. Denote by $H^{1}(\Omega)$ the standard Sobolev space, e.i., $H^{1}(\Omega)=\left\{v \in L^{2}(\Omega) \mid v_{x_{i}} \in L^{2}(\Omega), i=\overline{1, n}\right\}$ with scalar product $(v, w)_{H^{1}(\Omega)}=$ $\int_{\Omega}[\nabla v \nabla w+v w] d x$, where $\nabla u:=\left(u_{x_{1}}, \ldots, u_{x_{n}}\right), \nabla w:=\left(w_{x_{1}}, \ldots, w_{x_{n}}\right)$.

Let $K$ be a convex closed set in $H^{1}(\Omega)$ which contains 0 . Let us consider the problem of finding a function $u \in L_{\mathrm{loc}}^{2}(\bar{Q})$ such that $u_{x_{i}} \in L_{\mathrm{loc}}^{2}(\bar{Q}), i=\overline{1, n}$, $u_{t} \in L_{\mathrm{loc}}^{2}(\bar{Q})$, and, for a.e. $t \in(-\infty, 0], u(\cdot, t) \in K$ and

$$
\begin{aligned}
\int_{\Omega_{t}}\left\{u_{t}(v-u)+\nabla u \nabla(v-u)\right. & \left.+u(v-u)+(v-u) \int_{\Omega} b(x, y, t) u(y, t) d y\right\} d x \\
& \geq \int_{\Omega_{t}} f(v-u) d x \quad \forall v \in K
\end{aligned}
$$

\footnotetext{
* Department of Differential Equations, Ivan Franko National University of Lviv, 1, Universytetska St., Lviv, 79000, Ukraine, mm.bokalo@gmail.com

${ }^{\dagger}$ Department of Differential Equations, Ivan Franko National University of Lviv, 1, Universytetska St., Lviv, 79000, Ukraine, irusichka.skira@gmail.com

(C) M. M. Bokalo, I. V. Skira, 2019.
} 


$$
\lim _{t \rightarrow-\infty}\|u(\cdot, t)\|_{L^{2}(\Omega)}=0,
$$

where $f \in L_{\text {loc }}^{2}(\bar{Q}), b \in L^{\infty}(\Omega \times \Omega \times(-\infty, 0))$.

As it will be shown in the sequel, if

$$
f \in L^{2}(Q), \quad \operatorname{esssup}_{(x, y, t) \in \Omega \times \Omega \times(-\infty, 0]}|b(x, y, t)| \sqrt{\operatorname{mes}_{n} \Omega}<K,
$$

where $K>0$ is a constant from inequality $K\|v\|_{L^{2}(\Omega)} \leq\|v\|_{H^{1}(\Omega)}, \forall v \in H^{1}(\Omega)$, then this problem, which we call problem (1.1),(1.2), has unique solution.

We remark that problem $(1.1),(1.2)$ can be written in more abstract way. Indeed, after appropriate identification of functions and functionals, we have continuous and dense imbedding

$$
H^{1}(\Omega) \subset L^{2}(\Omega) \subset\left(H^{1}(\Omega)\right)^{\prime},
$$

where $\left(H^{1}(\Omega)\right)^{\prime}$ is dual to $H^{1}(\Omega)$ space. Clearly, for any $h \in L^{2}(\Omega)$ and $v \in H^{1}(\Omega)$ we have $\langle h, v\rangle=(h, v)$, where $\langle\cdot, \cdot\rangle$ is the notation for scalar product on dual pair $\left[\left(H^{1}(\Omega)\right)^{\prime}, H^{1}(\Omega)\right]$, and $(\cdot, \cdot)$ is the scalar product in $L^{2}(\Omega)$. Thus, we can use the notation $(\cdot, \cdot)$ instead of $\langle\cdot, \cdot\rangle$.

Now, we denote $S:=(-\infty, 0], V:=H^{1}(\Omega), H:=L^{2}(\Omega)$ and define an operator $A: V \rightarrow V^{\prime}$ as follows

$$
(A v, w)=\int_{\Omega}[\nabla v \nabla w+v w] d x, \quad v, w \in V .
$$

For all $t \in S$ define an operator $B(t, \cdot): H \rightarrow H$ as follows

$$
B(t, v)(\cdot)=\int_{\Omega} b(\cdot, y, t) v(y) d y, \quad v \in H
$$

Then problem (1.1),(1.2) can be rewritten as following: find a function $u \in$ $L_{\text {loc }}^{2}(S ; V)$ such that $u^{\prime} \in L_{\text {loc }}^{2}(S ; H)$, condition (1.2) holds, and, for a.e. $t \in S$, $u(t) \in K$ and

$$
\left(u^{\prime}(t)+A u(t)+B(t, u(t)), v-u(t)\right) \geq(f(t), v-u(t)) \quad \forall v \in K .
$$

Here $f \in L_{\text {loc }}^{2}(S ; H)$ is a given function.

We remark that variational inequality (1.3) can be written as a subdifferential inclusion. For this purpose we put $I_{K}(v):=0$ if $v \in K$, and $I_{K}(v):=+\infty$ if $v \in V \backslash K$, and also

$$
\Phi(v)=\frac{1}{2} \int_{\Omega}\left(|\nabla v|^{2}+|v|^{2}\right) d x+I_{K}(v), \quad v \in V .
$$

It is easy to verify that the functional $\Phi: V \rightarrow \mathbb{R} \cup\{+\infty\}$ is convex and semilower-continuous. By the known results (see, e.g., [22, p. 83]) it follows that the problem of finding a solution of variational inequality (1.3) can be written 
as such subdifferential inclusion: to find a function $u \in L_{\text {loc }}^{2}(S ; V)$ such that $u^{\prime} \in L_{\mathrm{loc}}^{2}(S ; H)$, condition (1.2) holds and, for a.e. $t \in S, u(t) \in D(\partial \Phi)$ and

$$
u^{\prime}(t)+\partial \Phi(u(t))+B(t, u(t)) \ni f(t) \text { in } H .
$$

The aim of this paper is to investigate problems for inclusions of type (1.4).

Problem without initial conditions or, in other words, the Fourier problem for evolution equations and inclusions arise in modeling different nonstationary processes in nature, that started a long time ago and initial conditions do not affect on them in the actual time moment. Thus, we can assume that the initial time is $-\infty$, while 0 is the final time, and initial conditions can be replaced with the behaviour of the solution as time variable turns to $-\infty$. Such problem appear in modeling in many fields of science such as ecology, economics, physics, cybernetics, etc. The research of the problem without initial conditions for the evolution equations and variational inequalities were conducted in the monographs $[16,18$, $22]$, the papers $[3,6-8,13,15,17,19,21]$, and others. In particular, R.E. Showalter in the paper [21] proved the existence of a unique solution $u \in \mathrm{e}^{2 \omega \cdot} H^{1}(S ; H)$, where $H$ is a Hilbert space, of the problem without initial condition

$$
u^{\prime}(t)+\mu u(t)+A(u(t)) \ni f(t), \quad t \in S,
$$

for $\omega+\mu>0$ and $f \in \mathrm{e}^{2 \omega \cdot} H^{1}(S ; H)$, in case when $A: H \rightarrow 2^{H}$ is maximal monotone operator such that $0 \in A(0)$. Moreover, if $A=\partial \varphi$, where $\varphi: H \rightarrow$ $\mathbb{R} \cup\{+\infty\}$ is proper, convex and lower-semi-continuous functional such that $\varphi(0)=$ $0=\inf \{\varphi(v): v \in H\}$, then this problem is uniquely solvable for each $\mu>0$, $f \in L^{2}(S ; H)$ and $\omega=0$.

As is well known the uniqueness of the solutions of problem without initial conditions for linear parabolic equations and variational inequalities is possible only under some restrictions on the behavior of solutions as time variable terns to $-\infty$. For the first time it was strictly justified by A.N. Tikhonov [23] in the case of heat equation. However, as it was shown by M.M. Bokalo [3], problem without initial conditions for some nonlinear parabolic equations has a unique solution in the class of functions without behavior restriction as time variable terns to $-\infty$. Similar result for evolutionary variational inequalities were also obtained in the paper [4].

Note that in inclusion (1.4) the unknown function can enter both in the differential part and in functional part. Previously, the Fourier problem for evolution integro-differential equations were studied in $[5,9,10]$ (see also references therein). Let us note that problems without initial conditions for variational inequalities or inclusions with functionals have not been considered in the literature, and this serves as one of the motivations for the study of such problems.

The outline of this paper is as follows. In Section 2, we give notation, definitions of needed function spaces and auxiliary results. In Section 3, we formulate the problem and main result. We prove the main result in Section 4 . 


\section{Preliminaries}

Set $S:=(-\infty, 0]$. Let $V$ and $H$ be separable Hilbert spaces with the scalar products $(\cdot, \cdot)_{V},(\cdot, \cdot)$ and norms $\|\cdot\|,|\cdot|$, respectively. Suppose that $V \subset H$ with dense, continuous and compact injection, i.e., the closure of $V$ in $H$ coincides with $H$, and there exists a constant $\lambda>0$ such that

$$
\lambda|v|^{2} \leq\|v\|^{2} \quad \text { for all } v \in V
$$

and for every sequence $\left\{v_{k}\right\}_{k=1}^{\infty}$ bounded in $V$ there exist an element $v \in V$ and a subsequence $\left\{v_{k_{j}}\right\}_{j=1}^{\infty}$ such that $v_{k_{j}} \underset{j \rightarrow \infty}{\longrightarrow} v$ strongly in $H$.

Let $V^{\prime}$ and $H^{\prime}$ be the dual spaces to $V$ and $H$, respectively. We suppose (after appropriate identification of functionals), that the space $H^{\prime}$ is a subspace of $V^{\prime}$. Identifying the spaces $H$ and $H^{\prime}$ by the Riesz-Fréchet representation theorem, we obtain dense and continuous embeddings

$$
V \subset H \subset V^{\prime} .
$$

Note that in this case $\langle g, v\rangle_{V}=(g, v)$ for every $v \in V, g \in H$, where $\langle\cdot, \cdot\rangle_{V}$ is the scalar product for the duality $\left[V^{\prime}, V\right]$. Therefore, further we can use the notation $(\cdot, \cdot)$ instead of $\langle\cdot, \cdot\rangle_{V}$.

We introduce some spaces of functions and distributions. Let $X$ be an arbitrary Hilbert space with the scalar product $(\cdot, \cdot)_{X}$ and the norm $\|\cdot\|_{X}$. By $C(S ; X)$ we mean the linear space of continuous functions defined on $S$ with values in $X$. We say that $w_{m} \underset{m \rightarrow \infty}{\longrightarrow} w$ in $C(S ; X)$ if for each $t_{1}, t_{2} \in S, t_{1}<t_{2}$, we have $\max _{t \in\left[t_{1}, t_{2}\right]}\left\|w(t)-w_{m}(t)\right\|_{X} \underset{m \rightarrow \infty}{\longrightarrow} 0$.

Denote by $L_{\text {loc }}^{2}(S ; X)$ the linear space of measurable functions defined on $S$ with values in $X$, whose restrictions to any segment $\left[t_{1}, t_{2}\right] \subset S$ belong to the space $L^{2}\left(t_{1}, t_{2} ; X\right)$. We say that a sequence $\left\{w_{m}\right\}$ is bounded (respectively, strongly, weakly or $*$-weakly convergent to $w)$ in $L_{\text {loc }}^{2}(S ; X)$, if for each $t_{1}, t_{2} \in S, t_{1}<t_{2}$, the sequence of restrictions of $\left\{w_{m}\right\}$ on the segment $\left[t_{1}, t_{2}\right]$ is bounded (respectively, strongly, weakly or $*$-weakly convergent to the restriction of $w$ on this segment) in $L^{2}\left(t_{1}, t_{2} ; X\right)$.

Let $\nu \in \mathbb{R}$. Put by definition

$$
L_{\nu}^{2}(S ; X):=\left\{f \in L_{\mathrm{loc}}^{2}(S ; X) \mid \int_{S} e^{2 \nu t}\|f(t)\|_{X}^{2} d t<\infty\right\} .
$$

This space is a Hilbert space with the scalar product

$$
(f, g)_{L_{\nu}^{2}(S ; X)}=\int_{S} e^{2 \nu t}(f(t), g(t))_{X} d t
$$

and the corresponding norm

$$
\|f\|_{L_{\nu}^{2}(S ; X)}:=\left(\int_{S} e^{2 \nu t}\|f(t)\|_{X}^{2} d t\right)^{1 / 2} .
$$


Also we introduce the space

$$
L_{\nu}^{\infty}(S ; X):=\left\{f \in L_{\mathrm{loc}}^{\infty}(S ; X) \mid \underset{t \in S}{\operatorname{ess} \sup }\left[e^{\nu t}\|f(t)\|_{X}\right]<\infty\right\} .
$$

By $D^{\prime}\left(-\infty, 0 ; V^{\prime}\right)$ we mean the space of continuous linear functionals on $D(-\infty, 0)$ with values in $V_{w}^{\prime}$ (hereafter $D(-\infty, 0)$ is space of test functions, that is, the space of infinitely differentiable on $(-\infty, 0)$ functions with compact supports, equipped with the corresponding topology, and $V_{w}^{\prime}$ is the linear space $V^{\prime}$ equipped with weak topology). It is easy to see (using (2.2)), that spaces $L_{\text {loc }}^{2}(S ; V)$, $L_{\text {loc }}^{2}(S ; H), L_{\text {loc }}^{2}\left(S ; V^{\prime}\right)$ can be identified with the corresponding subspaces of $D^{\prime}\left(-\infty, 0 ; V^{\prime}\right)$. In particular, this allows us to talk about derivatives $w^{\prime}$ of functions $w$ from $L_{\text {loc }}^{2}(S ; V)$ or $L_{\text {loc }}^{2}(S ; H)$ in the sense of distributions $D^{\prime}\left(-\infty, 0 ; V^{\prime}\right)$ and belonging of such derivatives to $L_{\mathrm{loc}}^{2}(S ; H)$ or $L_{\mathrm{loc}}^{2}\left(S ; V^{\prime}\right)$.

Let us define the spaces

$$
\begin{aligned}
H_{\mathrm{loc}}^{1}(S ; H) & :=\left\{w \in L_{\mathrm{loc}}^{2}(S ; H) \mid w^{\prime} \in L_{\mathrm{loc}}^{2}(S ; H)\right\}, \\
W_{2, \mathrm{loc}}(S ; V) & :=\left\{w \in L_{\mathrm{loc}}^{2}(S ; V) \mid w^{\prime} \in L_{\mathrm{loc}}^{2}\left(S ; V^{\prime}\right)\right\} .
\end{aligned}
$$

From known results (see., for example, [14, pp. 177-179]) it follows that

$$
H_{\mathrm{loc}}^{1}(S ; H) \subset C(S ; H) \quad \text { and } \quad W_{2, \mathrm{loc}}(S ; V) \subset C(S ; H) \text {. }
$$

Moreover, for every $w$ in $H_{\text {loc }}^{1}(S ; H)$ or $W_{2, \text { loc }}(S ; V)$ the function $t \rightarrow|w(t)|^{2}$ is absolutely continuous on any segment of the interval $S$ and the following equality holds

$$
\frac{d}{d t}|w(t)|^{2}=2\left(w^{\prime}(t), w(t)\right) \quad \text { for a.e. } \quad t \in S
$$

Denote

$$
H_{\nu}^{1}(S ; H):=\left\{w \in L_{\nu}^{2}(S ; H) \mid w^{\prime} \in L_{\nu}^{2}(S ; H)\right\}, \quad \nu \in \mathbb{R} .
$$

In this paper we use the following well-known facts.

Lemma 2.1 (Cauchy-Schwarz inequality [14, p. 158]). Suppose that $t_{1}, t_{2} \in \mathbb{R}$, $t_{1}<t_{2}$, and $X$ is a Hilbert space with the scalar product $(\cdot, \cdot)_{X}$. Then, for $v, w \in L^{2}\left(t_{1}, t_{2} ; X\right)$, we have $(w(\cdot), v(\cdot))_{X} \in L^{1}\left(t_{1}, t_{2}\right)$ and

$$
\int_{t_{1}}^{t_{2}}(w(t), v(t))_{X} d t \leq\|w\|_{L^{2}\left(t_{1}, t_{2} ; X\right)}\|v\|_{L^{2}\left(t_{1}, t_{2} ; X\right)} .
$$

Lemma 2.2 ( [27, pp. 173,179]). Let $Y$ be a Banach space with the norm $\|\cdot\|_{Y}$, and $\left\{v_{k}\right\}_{k=1}^{\infty}$ be a sequence of elements of $Y$, which is weakly or *-weakly convergent to $v$ in $Y$. Then $\varliminf_{k \rightarrow \infty}\left\|v_{k}\right\|_{Y} \geq\|v\|_{Y}$.

Lemma 2.3 (Aubin theorem [1], [2, p. 393]). Let $q>1, r>1, t_{1}, t_{2} \in \mathbb{R}, t_{1}<t_{2}$, and $\mathcal{W}, \mathcal{L}, \mathcal{B}$ are Banach spaces such that $\mathcal{W} \stackrel{c}{\subset} \mathcal{L} \circlearrowleft \mathcal{B}$ (here $\stackrel{c}{\subset}$ means compact embedding, and $\circlearrowleft$ means continuous embedding). Then

$$
\left\{w \in L^{q}\left(t_{1}, t_{2} ; \mathcal{W}\right) \mid w^{\prime} \in L^{r}\left(t_{1}, t_{2} ; \mathcal{B}\right)\right\} \stackrel{c}{\subset}\left(L^{q}\left(t_{1}, t_{2} ; \mathcal{L}\right) \cap C\left(\left[t_{1}, t_{2}\right] ; \mathcal{B}\right)\right) .
$$


Note that, we understand embedding (2.5) as follows: if a sequence $\left\{w_{m}\right\}$ is bounded in the space $L^{q}\left(t_{1}, t_{2} ; \mathcal{W}\right)$ and the sequence $\left\{w_{m}^{\prime}\right\}$ is bounded in the space $L^{r}\left(t_{1}, t_{2} ; \mathcal{B}\right)$, then there exist a function $w \in C\left(\left[t_{1}, t_{2}\right] ; \mathcal{B}\right) \cap L^{q}\left(t_{1}, t_{2} ; \mathcal{L}\right)$ and a subsequence $\left\{w_{m_{j}}\right\}$ of the sequence $\left\{w_{m}\right\}$ such that $w_{m_{j}} \underset{j \rightarrow \infty}{\longrightarrow} w$ in $C\left(\left[t_{1}, t_{2}\right] ; \mathcal{B}\right)$ and strongly in $L^{q}\left(t_{1}, t_{2} ; \mathcal{L}\right)$.

Lemma 2.4. If a sequence $\left\{w_{m}\right\}$ is bounded in the space $L_{\text {loc }}^{2}(S ; V)$ and the sequence $\left\{w_{m}^{\prime}\right\}$ is bounded in the space $L_{\text {loc }}^{2}(S ; H)$, then there exist a function $w \in L_{\mathrm{loc}}^{2}(S ; V), w^{\prime} \in L_{\mathrm{loc}}^{2}(S ; H)$, and a subsequence $\left\{w_{m_{j}}\right\}$ of the sequence $\left\{w_{m}\right\}$ such that $w_{m_{j}} \underset{j \rightarrow \infty}{\longrightarrow} w$ in $C(S ; H)$ and weakly in $L_{\mathrm{loc}}^{2}(S ; V)$, and $w_{m_{j}}^{\prime} \underset{j \rightarrow \infty}{\longrightarrow} w^{\prime}$ weakly in $L_{\mathrm{loc}}^{2}(S ; H)$.

Proof of Lemma 2.4. Lemma 2.3 for $q=2, r=2, \mathcal{W}=V, \mathcal{L}=\mathcal{B}=H$ and reflexiveness of Hilbert spaces yield, for every $t_{1}, t_{2} \in S, t_{1}<t_{2}$, from the sequence of restrictions of the elements $\left\{w_{m}\right\}$ to the segment $\left[t_{1}, t_{2}\right]$ one can choose a subsequence which is convergent in $C\left(\left[t_{1}, t_{2}\right] ; H\right)$ and weakly in $L^{2}\left(t_{1}, t_{2} ; V\right)$, and the sequence of derivatives of the elements of this subsequence is weakly convergent in $L^{2}\left(t_{1}, t_{2} ; H\right)$. For each $k \in \mathbb{N}$ we choose a subsequence $\left\{w_{m_{k, j}}\right\}_{j=1}^{\infty}$ of the given sequence which is convergent in $C([-k, 0] ; H)$ and weakly in $L^{2}(-k, 0 ; V)$ to some function $\widehat{w}_{k} \in C([-k, 0] ; H) \cap L^{2}(-k, 0 ; V)$, and the sequence $\left\{w_{m_{k, j}}^{\prime}\right\}_{j=1}^{\infty}$ is weakly convergent to the derivative $\widehat{w}_{k}^{\prime}$ in $L^{2}(-k, 0 ; H)$. Making this choice we ensure that the sequence $\left\{w_{m_{k+1, j}}\right\}_{j=1}^{\infty}$ was a subsequence of the sequence $\left\{w_{m_{k, j}}\right\}_{j=1}^{\infty}$. Now, according to the diagonal process we select the desired subsequence as $\left\{w_{m_{j, j}}\right\}_{j=1}^{\infty}$, and we define the function $w$ as follows: for each $k \in \mathbb{N}$ we take $w(t):=\widehat{w}_{k}(t)$ for $t \in(-k,-k+1]$.

\section{Statement of the problem and main result}

Let $\Phi: V \rightarrow \mathbb{R}_{\infty}:=(-\infty,+\infty]$ be a proper functional, i.e.,

$$
\operatorname{dom}(\Phi):=\{v \in V: \Phi(v)<+\infty\} \neq \emptyset
$$

which satisfies the conditions:

$$
\Phi(\alpha v+(1-\alpha) w) \leq \alpha \Phi(v)+(1-\alpha) \Phi(w) \quad \forall v, w \in V, \forall \alpha \in[0,1]
$$

i.e., the functional $\Phi$ is convex,

$$
v_{k} \underset{k \rightarrow \infty}{\longrightarrow} v \text { in } V \quad \Longrightarrow \quad \lim _{k \rightarrow \infty} \Phi\left(v_{k}\right) \geq \Phi(v),
$$

i.e., the functional $\Phi$ is lower semicontinuous. 
Recall that the subdifferential of functional $\Phi$ is a mapping $\partial \Phi: V \rightarrow 2^{V^{\prime}}$, defined as follows

$$
\partial \Phi(v):=\left\{v^{*} \in V^{\prime} \mid \Phi(w) \geq \Phi(v)+\left(v^{*}, w-v\right) \quad \forall w \in V\right\}, \quad v \in V,
$$

and the domain of the subdifferential $\partial \Phi$ is the set $D(\partial \Phi):=\{v \in V \mid \partial \Phi(v) \neq \emptyset\}$. We identify the subdifferential $\partial \Phi$ with its graph, assuming that $\left[v, v^{*}\right] \in \partial \Phi$ if and only if $v^{*} \in \partial \Phi(v)$, i.e., $\left.\partial \Phi=\left\{\left[v, v^{*}\right] \mid v \in D(\partial \Phi), v^{*} \in \partial \Phi(v)\right)\right\}$. R. Rockafellar in paper [20, Theorem A] proves that the subdifferential $\partial \Phi$ is a maximal monotone operator, that is,

$$
\left(v_{1}^{*}-v_{2}^{*}, v_{1}-v_{2}\right) \geq 0 \quad \forall\left[v_{1}, v_{1}^{*}\right],\left[v_{2}, v_{2}^{*}\right] \in \partial \Phi,
$$

and for every element $\left[v_{1}, v_{1}^{*}\right] \in V \times V^{\prime}$ we have the implication

$$
\left(v_{1}^{*}-v_{2}^{*}, v_{1}-v_{2}\right) \geq 0 \quad \forall\left[v_{2}, v_{2}^{*}\right] \in \partial \Phi \quad \Longrightarrow \quad\left[v_{1}, v_{1}^{*}\right] \in \partial \Phi .
$$

Let, for each $t \in S, B(t, \cdot): H \rightarrow H$ be an operator which satisfies the condition:

$(\mathcal{B})$ for any $v \in H$ the mapping $B(\cdot, v): S \rightarrow S$ is measurable, and there exists a constant $L \geq 0$ such that following inequality holds

$$
\left|B\left(t, v_{1}\right)-B\left(t, v_{2}\right)\right| \leq L\left|v_{1}-v_{2}\right|
$$

for a.e. $t \in S$, and for all $v_{1}, v_{2} \in H$; in addition, $B(t, 0)=0$ for a.e. $t \in S$.

Remark 3.1. From the condition $(\mathcal{B})$ it follows that for a.e. $t \in S$, and for every $v \in H$ the following estimate is valid:

$$
|B(t, v)| \leq L|v|
$$

Let us consider the evolutionary variational inequality

$$
u^{\prime}(t)+\partial \Phi(u(t))+B(t, u(t)) \ni f(t), \quad t \in S,
$$

where $f: S \rightarrow V^{\prime}$ is a given measurable function and $u: S \rightarrow V$ is an unknown function.

Definition 3.1. Let conditions $\left(\mathcal{A}_{1}\right),\left(\mathcal{A}_{2}\right),(\mathcal{B})$ hold, and $f \in L_{\text {loc }}^{2}\left(S ; V^{\prime}\right)$. The solution of variational inequality (3.3) is a function $u: S \rightarrow V$ that satisfies the following conditions:

1) $u \in W_{2, \operatorname{loc}}(S ; V)$;

2) $u(t) \in D(\partial \Phi)$ for a.e. $t \in S$; 
3) there exists a function $g \in L_{\text {loc }}^{2}\left(S ; V^{\prime}\right)$ such that, for a.e. $t \in S, g(t) \in$ $\partial \Phi(u(t))$ and

$$
u^{\prime}(t)+g(t)+B(t, u(t))=f(t) \quad \text { in } V^{\prime} .
$$

For variational inequality (3.3) consider the problem: find its solution which satisfies the condition

$$
\lim _{t \rightarrow-\infty} e^{\gamma t}|u(t)|=0
$$

where $\gamma \in \mathbb{R}$ is given.

The problem of finding a solution of variational inequality (3.3) (for given $\Phi, B, f)$ satisfying the condition (3.4) for given $\gamma$, is called the Fourier problem or, in other words, the problem without initial conditions for the evolution variational inequality (3.3). This problem, in short, be called the problem $\mathbf{P}(\Phi, B, f, \gamma)$, and the function $u$ is called its solution.

Additionally, assume that the following conditions hold:

$\left(\mathcal{A}_{3}\right)$ there exists a constant $K_{1}>0$ such that

$$
\left(v_{1}^{*}-v_{2}^{*}, v_{1}-v_{2}\right) \geq K_{1}\left|v_{1}-v_{2}\right|^{2} \quad \forall\left[v_{1}, v_{1}^{*}\right],\left[v_{2}, v_{2}^{*}\right] \in \partial \Phi ;
$$

$\left(\mathcal{A}_{4}\right)$ there exists a constant $K_{2}>0$ such that

$$
\Phi(v) \geq K_{2}\|v\|^{2} \quad \forall v \in \operatorname{dom}(\Phi) ;
$$

moreover, $\Phi(0)=0$.

Remark 3.2. Condition $\left(\mathcal{A}_{4}\right)$ implies that $\Phi(v) \geq \Phi(0)+(0, v-0) \forall v \in V$, hence $[0,0] \in \partial \Phi$. From this and condition $\left(\mathcal{A}_{3}\right)$ we have

$$
\left(v^{*}, v\right) \geq K_{1}|v|^{2} \quad \forall\left[v, v^{*}\right] \in \partial \Phi .
$$

Now we shall formulate the main result.

Theorem 3.1. Let conditions $\left(\mathcal{A}_{1}\right)-\left(\mathcal{A}_{3}\right),(\mathcal{B})$ hold, and $\gamma \in \mathbb{R}$ is such that

$$
\gamma<K_{1}-L \text {. }
$$

Then the problem $\boldsymbol{P}(\Phi, B, f, \gamma)$ has at most one solution.

Theorem 3.2. Let conditions $\left(\mathcal{A}_{1}\right)-\left(\mathcal{A}_{4}\right),(\mathcal{B})$ hold, and

$(\mathcal{F}) \quad f \in L_{\gamma}^{2}(S ; H)$,

where $\gamma \in \mathbb{R}$ satisfies inequality (3.6). Then the problem $\boldsymbol{P}(\Phi, B, f, \gamma)$ has a unique solution, it belongs to the space $L_{\gamma}^{\infty}(S ; V) \cap L_{\gamma}^{2}(S ; V) \cap H_{\gamma}^{1}(S ; H)$ and satisfies the estimate:

$$
\begin{aligned}
e^{2 \gamma \sigma}\|u(\sigma)\|^{2} & +\int_{-\infty}^{\sigma} e^{2 \gamma t}\|u(t)\|^{2} d t+\int_{-\infty}^{\sigma} e^{2 \gamma t}\left|u^{\prime}(t)\right|^{2} d t \\
& +\int_{-\infty}^{\sigma} e^{2 \gamma t} \Phi(u(t)) d t \leq C_{1} \int_{-\infty}^{\sigma} e^{2 \gamma t}|f(t)|^{2} d t, \quad \sigma \in S,
\end{aligned}
$$

where $C_{1}$ is a positive constant depending on $K_{1}, K_{2}, L$ and $\gamma$ only. 
Remark 3.3. The problem $\mathbf{P}(\Phi, B, f, \gamma)$ can be replaced by the following problem. Let $K$ be a convex and closed set in $V, A: V \rightarrow V^{\prime}$ be a monotone, bounded and semi-continuous operator such that $(A(v), v) \geq \widetilde{K}_{1}\|v\|^{2} \quad \forall v \in V$, where $\widetilde{K}_{1}=$ const $>0$. The problem is to find a function $u \in W_{2, \text { loc }}(S ; V)$ satisfying the condition (3.4) and, for a.e. $t \in S, u(t) \in K$ and

$$
\left(u^{\prime}(t)+A(u(t))+B(t, u(t)), v-u(t)\right) \geq(f(t), v-u(t)) \quad \forall v \in K .
$$

\section{Proof of the main result}

Proof of the Theorem 3.1. Assume the contrary. Let $u_{1}, u_{2}$ be two solutions of the problem $\mathbf{P}(\Phi, B, f, \gamma)$. Then for every $i \in\{1,2\}$ there exists function $g_{i} \in L_{\mathrm{loc}}^{2}\left(S ; V^{\prime}\right)$ such that, for a.e. $t \in S, g_{i}(t) \in \partial \Phi\left(u_{i}(t)\right)$ and

$$
u_{i}^{\prime}(t)+g_{i}(t)+B\left(t, u_{i}(t)\right)=f(t) \quad \text { in } V^{\prime} .
$$

We put $w(t):=u_{1}(t)-u_{2}(t), t \in S$. From equalities (4.1) for a.e. $t \in S$ we obtain

$$
w^{\prime}(t)+g_{1}(t)-g_{2}(t)+B\left(t, u_{1}(t)\right)-B\left(t, u_{2}(t)\right)=0 \quad \text { in } V^{\prime} .
$$

From (3.4) it follows that the following condition holds

$$
e^{2 \gamma t}|w(t)|^{2} \rightarrow 0 \quad \text { as } \quad t \rightarrow-\infty .
$$

Let $\sigma_{1}, \sigma_{2} \in S$ be arbitrary numbers such that $\sigma_{1}<\sigma_{2}$. Multiplying equality (4.2) by $w(t) e^{2 \gamma t}$, and integrating from $\sigma_{1}$ to $\sigma_{2}$ we obtain

$$
\begin{aligned}
\int_{\sigma_{1}}^{\sigma_{2}} e^{2 \gamma t}\left(w^{\prime}(t), w(t)\right) d t & +\int_{\sigma_{1}}^{\sigma_{2}} e^{2 \gamma t}\left(g_{1}(t)-g_{2}(t), u_{1}(t)-u_{2}(t)\right) d t \\
& +\int_{\sigma_{1}}^{\sigma_{2}} e^{2 \gamma t}\left(B\left(t, u_{1}(t)\right)-B\left(t, u_{2}(t)\right), w(t)\right) d t=0 .
\end{aligned}
$$

By condition $\left(\mathcal{A}_{3}\right)$ and the fact that $g_{i}(t) \in \partial \Phi\left(u_{i}(t)\right), i=1,2$, for a.e. $t \in S$ we have the inequality

$$
\left(g_{1}(t)-g_{2}(t), u_{1}(t)-u_{2}(t)\right) \geq K_{1}|w(t)|^{2} .
$$

Consider the last term from left-hand side of equality (4.4). Using (3.1) and the Cauchy-Schwarz inequality, we have

$$
\begin{aligned}
\mid \int_{\sigma_{1}}^{\sigma_{2}} e^{2 \gamma t}\left(B\left(t, u_{1}(t)\right)\right. & \left.-B\left(t, u_{2}(t)\right), w(t)\right) d t \mid \\
& \leq \int_{\sigma_{1}}^{\sigma_{2}} e^{2 \gamma t}\left|B\left(t, u_{1}(t)\right)-B\left(t, u_{2}(t)\right)\right||w(t)| d t \\
& \leq L \int_{\sigma_{1}}^{\sigma_{2}} e^{2 \gamma t}|w(t)|^{2} d t .
\end{aligned}
$$


By (2.3), (4.5), (4.6), from (4.4) we obtain the following inequality

$$
\frac{1}{2} \int_{\sigma_{1}}^{\sigma_{2}} e^{2 \gamma t} \frac{d|w(t)|^{2}}{d t} d t+\left(K_{1}-L\right) \int_{\sigma_{1}}^{\sigma_{2}} e^{2 \gamma t}|w(t)|^{2} d t \leq 0 .
$$

Using the integration-by-parts formula, from (4.7) we have

$$
\left.e^{2 \gamma t}|w(t)|^{2}\right|_{\sigma_{1}} ^{\sigma_{2}}+2\left(K_{1}-L-\gamma\right) \int_{\sigma_{1}}^{\sigma_{2}} e^{2 \gamma t}|w(t)|^{2} d t \leq 0
$$

Since condition (3.6) holds, from (4.8) we obtain

$$
e^{2 \gamma \sigma_{2}}\left|w\left(\sigma_{2}\right)\right|^{2} \leq e^{2 \gamma \sigma_{1}}\left|w\left(\sigma_{1}\right)\right|^{2}
$$

Let us fix an arbitrary $\sigma_{2}$ in (1.1), and pass to the limit as $\sigma_{1} \rightarrow-\infty$. According to condition (4.3), the the right side of inequality (1.1) turns to 0 . Thus, we get the equality $e^{2 \gamma \sigma_{2}}\left|w\left(\sigma_{2}\right)\right|^{2}=0$. Since $\sigma_{2} \in S$ is an arbitrary number, we have $w(t)=0$ for a.e. $t \in S$, this contradicts our assumption. Therefore, a solution of the problem $\mathbf{P}(\Phi, B, f, \gamma)$ is unique.

Proof of the Theorem 3.2. We divide the proof into five steps.

Step 1 (auxiliary statements). Under assumptions $\left(\mathcal{A}_{1}\right),\left(\mathcal{A}_{2}\right)$ we define the functional $\Phi_{H}: H \rightarrow \mathbb{R}_{\infty}$ by the rule: $\Phi_{H}(v):=\Phi(v)$, if $v \in V$, and $\Phi_{H}(v):=+\infty$ otherwise. Note that conditions $\left(\mathcal{A}_{1}\right),\left(\mathcal{A}_{2}\right)$, Lemma IV.5.2 and Proposition IV.5.2 of the monograph [22] imply that $\Phi_{H}$ is a proper, convex and lower-semi-continuous functional on $H$, $\operatorname{dom}\left(\Phi_{H}\right)=\operatorname{dom}(\Phi) \subset V$ and $\partial \Phi_{H}=$ $\partial \Phi \cap(V \times H)$, where $\partial \Phi_{H}: H \rightarrow 2^{H}$ is the subdifferential of the functional $\Phi_{H}$.

The following statements will be used in the sequel.

Lemma 4.1 ( [22, Lemma IV.4.3]). Let $-\infty<a<b<+\infty, w \in H^{1}(a, b ; H)$, and there exists $g \in L^{2}(a, b ; H)$ such that $g(t) \in \partial \Phi_{H}(w(t))$ for a.e. $t \in(a, b)$. Then the function $\Phi_{H}(w(\cdot))$ is absolutely continuous on the interval $[a, b]$ and for any function $h:[a, b] \rightarrow H$ such that $h(t) \in \partial \Phi_{H}(w(t))$ the following equality holds

$$
\frac{d}{d t} \Phi_{H}(w(t))=\left(h(t), w^{\prime}(t)\right) \quad \text { for a.e. } t \in(a, b) .
$$

Lemma 4.2 ( [11, Proposition 3.12], [22, Proposition IV.5.2]). Let T $>0, \tilde{f} \in$ $L^{2}(0, T ; H)$ and $w_{0} \in \operatorname{dom}(\Phi)$. Then there exists a unique function

$$
w \in C([0, T] ; H) \cap H^{1}(0, T ; H)
$$

such that $w(0)=w_{0}$ and, for a.e. $t \in(0, T], w(t) \in D\left(\partial \Phi_{H}\right)$ and

$$
w^{\prime}(t)+\partial \Phi_{H}(w(t)) \ni \tilde{f}(t) \quad \text { in } H .
$$


Lemma 4.3. Let $t_{0}<0, \tilde{f} \in L^{2}\left(t_{0}, 0 ; H\right)$, and $w_{0} \in \operatorname{dom}(\Phi)$. Then there exists a unique function $w \in C\left(\left[t_{0}, 0\right] ; H\right) \cap H^{1}\left(t_{0}, 0 ; H\right)$ such that $w\left(t_{0}\right)=w_{0}$ and, for a.e. $t \in\left(t_{0}, 0\right], w(t) \in D\left(\partial \Phi_{H}\right)$ and

$$
w^{\prime}(t)+\partial \Phi_{H}(w(t))+B(t, w(t)) \ni \widetilde{f}(t) \quad \text { in } H,
$$

that is, there exists $\widetilde{g} \in L^{2}\left(t_{0}, 0 ; H\right)$ such that, for a.e. $t \in\left(t_{0}, 0\right]$, we have $\widetilde{g}(t) \in \partial \Phi_{H}(w(t))$ and

$$
w^{\prime}(t)+\widetilde{g}(t)+B(t, w(t))=\widetilde{f}(t) \quad \text { in } H .
$$

Proof of Lemma 4.3. Let $\alpha>0$ be an arbitrary fixed number and set

$$
M:=\left\{w \in C\left(\left[t_{0}, 0\right] ; H\right) \mid w\left(t_{0}\right)=w_{0}\right\} .
$$

Consider $M$ with the metric

$$
\rho\left(w_{1}, w_{2}\right)=\max _{t \in\left[t_{0}, 0\right]}\left[e^{-\alpha\left(t-t_{0}\right)}\left|w_{1}(t)-w_{2}(t)\right|\right], \quad w_{1}, w_{2} \in M .
$$

It is obvious that the metric space $(M, \rho)$ is complete. Now let us consider an operator $A: M \rightarrow M$ defined as follows: for any given function $\widetilde{w} \in M$, it defines a function $\widehat{w} \in M \cap H^{1}\left(t_{0}, 0 ; H\right)$ such that, for a.e. $t \in\left(t_{0}, 0\right], \widehat{w}(t) \in D\left(\partial \Phi_{H}\right)$ and

$$
\widehat{w}^{\prime}(t)+\partial \Phi_{H}(\widehat{w}(t)) \ni \widetilde{f}(t)-B(t, \widetilde{w}(t)) \text { in } H .
$$

Clearly, variational inequality (4.13) coincides with variational inequality (4.10) after replacing $[0, T]$ by $\left[t_{0}, 0\right], \widetilde{f}(t)$ by $\widetilde{f}(t)-B(t, \widetilde{w}(t))$, the condition $w(0)=w_{0}$ by the condition $\widehat{w}\left(t_{0}\right)=w_{0}$. Thus, using Lemma 4.2 , we get that operator $A$ is well-defined. Let us show that the operator $A$ is a contraction for some $\alpha>0$. Indeed, let $\widetilde{w}_{1}, \widetilde{w}_{2}$ be arbitrary functions from $M$ and $\widehat{w}_{1}:=A \widetilde{w}_{1}, \widehat{w}_{2}:=A \widetilde{w}_{2}$. According to (4.13) there exist functions $\widehat{g}_{1}$ and $\widehat{g}_{2}$ from $L^{2}\left(t_{0}, 0 ; H\right)$ such that for every $k \in\{1,2\}$ and for a.e. $t \in\left(t_{0}, 0\right]$ we have $\widehat{g}_{k}(t) \in \partial \Phi_{H}\left(\widehat{w}_{k}(t)\right)$ and

$$
\widehat{w}_{k}^{\prime}(t)+\widehat{g}_{k}(t)=\widetilde{f}(t)-B\left(t, \widetilde{w}_{k}(t)\right)
$$

while $\widehat{w}_{k}\left(t_{0}\right)=w_{0}$.

Subtracting identity (4.14) for $k=2$ from identity (4.14) for $k=1$, and, for a.e. $t \in\left(t_{0}, 0\right]$, multiplying the obtained identity by $\widehat{w}_{1}(t)-\widehat{w}_{2}(t)$, we get

$$
\begin{gathered}
\left(\left(\widehat{w}_{1}(t)-\widehat{w}_{2}(t)\right)^{\prime}, \widehat{w}_{1}(t)-\widehat{w}_{2}(t)\right)+\left(\widehat{g}_{1}(t)-\widehat{g}_{2}(t), \widehat{w}_{1}(t)-\widehat{w}_{2}(t)\right) \\
=-\left(B\left(t, \widetilde{w}_{1}(t)\right)-B\left(t, \widetilde{w}_{2}(t)\right), \widehat{w}_{1}(t)-\widehat{w}_{2}(t)\right) \text { for a.e. } t \in\left(t_{0}, 0\right], \\
\widehat{w}_{1}\left(t_{0}\right)-\widehat{w}_{2}\left(t_{0}\right)=0 .
\end{gathered}
$$

We integrate equality (4.15) by $t$ from $t_{0}$ to $\sigma \in\left(t_{0}, 0\right]$, taking into account that for a.e. $t \in\left(t_{0}, 0\right]$ we have

$$
\left(\left(\widehat{w}_{1}(t)-\widehat{w}_{2}(t)\right)^{\prime}, \widehat{w}_{1}(t)-\widehat{w}_{2}(t)\right)=\frac{1}{2} \frac{d}{d t}\left|\widehat{w}_{1}(t)-\widehat{w}_{2}(t)\right|^{2} .
$$


As a result we get the equality

$$
\begin{aligned}
\frac{1}{2}\left|\widehat{w}_{1}(\sigma)-\widehat{w}_{2}(\sigma)\right|^{2} & +\int_{t_{0}}^{\sigma}\left(\widehat{g}_{1}(t)-\widehat{g}_{2}(t), \widehat{w}_{1}(t)-\widehat{w}_{2}(t)\right) d t \\
& =-\int_{t_{0}}^{\sigma}\left(B\left(t, \widetilde{w}_{1}(t)\right)-B\left(t, \widetilde{w}_{2}(t)\right), \widehat{w}_{1}(t)-\widehat{w}_{2}(t)\right) d t .
\end{aligned}
$$

By condition $\left(\mathcal{A}_{3}\right)$, for a.e. $t \in\left(t_{0}, 0\right]$ we have the inequality

$$
\left(\widehat{g}_{1}(t)-\widehat{g}_{2}(t), \widehat{w}_{1}(t)-\widehat{w}_{2}(t)\right) \geq K_{1}\left|\widehat{w}_{1}(t)-\widehat{w}_{2}(t)\right|^{2} .
$$

Taking into account condition $(\mathcal{B})$ and the Cauchy inequality, for a.e. $t \in\left(t_{0}, 0\right]$ we obtain

$$
\begin{aligned}
\mid\left(B\left(t, \widetilde{w}_{1}(t)\right)-B\left(t, \widetilde{w}_{2}(t)\right)\right. & \left., \widehat{w}_{1}(t)-\widehat{w}_{2}(t)\right) \mid \\
& \leq\left|B\left(t, \widetilde{w}_{1}(t)\right)-B\left(t, \widetilde{w}_{2}(t)\right)\right| \cdot\left|\widehat{w}_{1}(t)-\widehat{w}_{2}(t)\right| \\
& \leq L\left|\widetilde{w}_{1}(t)-\widetilde{w}_{2}(t)\right| \cdot\left|\widehat{w}_{1}(t)-\widehat{w}_{2}(t)\right| \\
& \leq \varepsilon\left|\widehat{w}_{1}(t)-\widehat{w}_{2}(t)\right|^{2}+\frac{L^{2}}{4 \varepsilon}\left|\widetilde{w}_{1}(t)-\widetilde{w}_{2}(t)\right|,
\end{aligned}
$$

where $\varepsilon>0$ is an arbitrary.

From (4.17), according to (4.18) and (4.19), we have

$$
\begin{aligned}
\left|\widehat{w}_{1}(\sigma)-\widehat{w}_{2}(\sigma)\right|^{2}+ & 2\left(K_{1}-\varepsilon\right) \int_{t_{0}}^{\sigma}\left|\widehat{w}_{1}(t)-\widehat{w}_{2}(t)\right|^{2} d t \\
& \leq(2 \varepsilon)^{-1} L^{2} \int_{t_{0}}^{\sigma}\left|\widetilde{w}_{1}(t)-\widetilde{w}_{2}(t)\right|^{2} d t .
\end{aligned}
$$

Choosing $\varepsilon=2^{-1} K_{1}$, from (4.20) we obtain

$$
\left|\widehat{w}_{1}(\sigma)-\widehat{w}_{2}(\sigma)\right|^{2} \leq C_{2} \int_{t_{0}}^{\sigma}\left|\widetilde{w}_{1}(t)-\widetilde{w}_{2}(t)\right|^{2} d t, \quad \sigma \in\left(t_{0}, 0\right],
$$

where $C_{2}>0$ is the constant.

After multiplying inequality (4.21) by $e^{-2 \alpha\left(\sigma-t_{0}\right)}$ we obtain

$$
\begin{aligned}
& e^{-2 \alpha\left(\sigma-t_{0}\right)}\left|\widehat{w}_{1}(\sigma)-\widehat{w}_{2}(\sigma)\right|^{2} \\
& \leq C_{2} e^{-2 \alpha\left(\sigma-t_{0}\right)} \int_{t_{0}}^{\sigma} e^{2 \alpha\left(t-t_{0}\right)} e^{-2 \alpha\left(t-t_{0}\right)}\left|\widetilde{w}_{1}(t)-\widetilde{w}_{2}(t)\right|^{2} d t \\
& \leq C_{2} e^{-2 \alpha\left(\sigma-t_{0}\right)} \max _{t \in\left[t_{0}, 0\right]}\left[-e^{\alpha\left(t-t_{0}\right)}\left|\widetilde{w}_{1}(t)-\widetilde{w}_{2}(t)\right|\right]^{2} \int_{t_{0}}^{\sigma} e^{2 \alpha\left(t-t_{0}\right)} d t \\
& =\frac{C_{2}}{2 \alpha}\left(1-e^{-2 \alpha\left(\sigma-t_{0}\right)}\right)\left[\rho\left(\widetilde{w}_{1}, \widetilde{w}_{2}\right)\right]^{2} \leq \frac{C_{2}}{2 \alpha}\left[\rho\left(\widetilde{w}_{1}, \widetilde{w}_{2}\right)\right]^{2}, \quad \sigma \in\left(t_{0}, 0\right] .
\end{aligned}
$$

From (4.22) it easily follows that

$$
\rho\left(\widehat{w}_{1}, \widehat{w}_{2}\right) \leq \sqrt{C_{2} /(2 \alpha)} \rho\left(\widetilde{w}_{1}, \widetilde{w}_{2}\right) .
$$


From this, choosing $\alpha>0$ such that inequality $C_{2} /(2 \alpha)<1$ holds, we obtain that operator $A$ is a contraction. Hence, we may apply the Banach fixed-point theorem [12, Theorem 5.7] and deduce that there exists a unique function $w \in M$ such that $A w=w$, i.e., we have proved Lemma 4.3.

Step 2 (solution approximation). We construct a sequence of functions which, in some sense, approximate the solution of the problem $\mathbf{P}(\Phi, B, f, \gamma)$.

For each $k \in \mathbb{N}$, let $\widehat{f}_{k}(t):=f(t)$ for $t \in S_{k}:=(-k, 0]$ and let us consider the problem of finding a function $\widehat{u}_{k} \in C\left(\overline{S_{k}} ; H\right) \cap H^{1}\left(S_{k} ; H\right)$, where $H^{1}\left(S_{k} ; H\right):=$ $\left\{w \in L^{2}\left(S_{k} ; H\right) \mid w^{\prime} \in L^{2}\left(S_{k} ; H\right)\right\}$, such that, for a.e. $t \in S_{k}$, we have $\widehat{u}_{k}(t) \in$ $D\left(\partial \Phi_{H}\right)$ and

$$
\begin{gathered}
\widehat{u}_{k}^{\prime}(t)+\partial \Phi_{H}\left(\widehat{u}_{k}(t)\right)+B\left(t, \widehat{u}_{k}(t)\right) \ni \widehat{f}_{k}(t) \text { in } H, \\
\widehat{u}_{k}(-k)=0 .
\end{gathered}
$$

Inclusion (4.23) means that there exists a function $\widehat{g}_{k} \in L^{2}\left(S_{k} ; H\right)$ such that, for a.e. $t \in S_{k}$, we have $\widehat{g}_{k}(t) \in \partial \Phi_{H}\left(\widehat{u}_{k}(t)\right)$ and

$$
\widehat{u}_{k}^{\prime}(t)+\widehat{g}_{k}(t)+B\left(t, \widehat{u}_{k}(t)\right)=\widehat{f}_{k}(t) \text { in } H .
$$

Since $D\left(\partial \Phi_{H}\right) \subset \operatorname{dom}\left(\Phi_{H}\right) \subset V$, thus $\widehat{u}_{k}(t) \in V$ for a.e. $t \in S_{k}$. According to the definition of the subdifferential of a functional and the fact that $\widehat{g}_{k}(t) \in$ $\partial \Phi\left(\widehat{u}_{k}(t)\right)$ for a.e. $t \in S_{k}$, we have

$$
\Phi(0) \geq \Phi\left(\widehat{u}_{k}(t)\right)+\left(\widehat{g}_{k}(t), 0-\widehat{u}_{k}(t)\right) \quad \text { for a.e. } \quad t \in S_{k} .
$$

Using this and condition $\left(\mathcal{A}_{4}\right)$ we obtain

$$
\left(\widehat{g}_{k}(t), \widehat{u}_{k}(t)\right) \geq \Phi\left(\widehat{u}_{k}(t)\right) \geq K_{2}\left\|\widehat{u}_{k}(t)\right\|^{2} \quad \text { for a.e. } \quad t \in S_{k} .
$$

Since the left side of this chain of inequalities belongs to $L^{1}\left(S_{k}\right)$, then $\widehat{u}_{k}$ belongs to $L^{2}\left(S_{k} ; V\right)$.

For each $k \in \mathbb{N}$ we extend functions $\widehat{f}_{k}, \widehat{u}_{k}$ and $\widehat{g}_{k}$ by zero for the entire interval $S$, and denote these extensions by $f_{k}, u_{k}$ and $g_{k}$ respectively. From the above it follows that, for each $k \in \mathbb{N}$, the function $u_{k}$ belongs to $L^{2}(S ; V)$, its derivative $u_{k}^{\prime}$ belongs to $L^{2}(S ; H)$ and, for a.e. $t \in S$, the inclusion $g_{k}(t) \in \partial \Phi_{H}\left(u_{k}(t)\right)$ and the following equality (see (4.25)) hold

$$
u_{k}^{\prime}(t)+g_{k}(t)+B\left(t, u_{k}(t)\right)=f_{k}(t) \quad \text { in } \quad H
$$

In order to show the convergence $\left\{u_{k}\right\}_{k=1}^{\infty}$ to the solution of the problem $\mathbf{P}(\Phi, B, f, \gamma)$ we need some estimates of the functions $u_{k}, k \in \mathbb{N}$.

Step 3 (estimates of solution approximations). 
Let $\sigma_{1}, \sigma_{2} \in S$ be arbitrary numbers such that $\sigma_{1}<\sigma_{2}$, and $k \in \mathbb{N}$. Multiplying identity (4.27), for a.e. $t \in S$, by $e^{2 \gamma t} u_{k}(t)$ and integrating from $\sigma_{1}$ to $\sigma_{2}$, we obtain

$$
\begin{aligned}
\int_{\sigma_{1}}^{\sigma_{2}} e^{2 \gamma t}\left(u_{k}^{\prime}(t), u_{k}(t)\right) d t+ & \int_{\sigma_{1}}^{\sigma_{2}} e^{2 \gamma t}\left(g_{k}(t), u_{k}(t)\right) d t \\
& +\int_{\sigma_{1}}^{\sigma_{2}} e^{2 \gamma t}\left(B\left(t, u_{k}(t)\right), u_{k}(t)\right) d t \\
= & \int_{\sigma_{1}}^{\sigma_{2}} e^{2 \gamma t}\left(f_{k}(t), u_{k}(t)\right) d t
\end{aligned}
$$

From this taking into account (2.3) and using the integration-by-parts formula, we obtain

$$
\begin{aligned}
& \left.e^{2 \gamma t}\left|u_{k}(t)\right|^{2}\right|_{\sigma_{1}} ^{\sigma_{2}}-2 \gamma \int_{\sigma_{1}}^{\sigma_{2}} e^{2 \gamma t}\left|u_{k}(t)\right|^{2} d t+2 \int_{\sigma_{1}}^{\sigma_{2}} e^{2 \gamma t}\left(g_{k}(t), u_{k}(t)\right) d t \\
& +2 \int_{\sigma_{1}}^{\sigma_{2}} e^{2 \gamma t}\left(B\left(t, u_{k}(t)\right), u_{k}(t)\right) d t=2 \int_{\sigma_{1}}^{\sigma_{2}} e^{2 \gamma t}\left(f_{k}(t), u_{k}(t)\right) d t .
\end{aligned}
$$

Acccording to the definition of $u_{k}$ and (4.26), we obtain

$$
\left(g_{k}(t), u_{k}(t)\right) \geq \Phi\left(u_{k}(t)\right) \geq K_{2}\left\|u_{k}(t)\right\|^{2} \quad \text { for a.e. } t \in S .
$$

Let us estimate the third term on the left-hand side of inequality (4.28). From (3.5) and (4.29) for arbitrary $\delta \in(0,1)$ we obtain

$$
\begin{aligned}
\int_{\sigma_{1}}^{\sigma_{2}} e^{2 \gamma t}\left(g_{k}(t), u_{k}(t)\right) d t= & (\delta+(1-\delta)) \int_{\sigma_{1}}^{\sigma_{2}} e^{2 \gamma t}\left(g_{k}(t), u_{k}(t)\right) d t \\
\geq & \delta K_{1} \int_{\sigma_{1}}^{\sigma_{2}} e^{2 \gamma t}\left|u_{k}(t)\right|^{2} d t \\
& +2^{-1}(1-\delta) K_{2} \int_{\sigma_{1}}^{\sigma_{2}} e^{2 \gamma t}\left\|u_{k}(t)\right\|^{2} d t \\
& +2^{-1}(1-\delta) \int_{\sigma_{1}}^{\sigma_{2}} e^{2 \gamma t} \Phi\left(u_{k}(t)\right) d t
\end{aligned}
$$

Now, let us estimate the last item on the left-hand side of inequality (4.28). Using the Cauchy-Shwarz inequality, (3.2) we have

$$
\begin{aligned}
\left|\int_{\sigma_{1}}^{\sigma_{2}} e^{2 \gamma t}\left(B\left(t, u_{k}(t)\right), u_{k}(t)\right) d t\right| & \leq \int_{\sigma_{1}}^{\sigma_{2}} e^{2 \gamma t}\left|B\left(t, u_{k}(t)\right)\right|\left|u_{k}(t)\right| d t \\
& \leq L \int_{\sigma_{1}}^{\sigma_{2}} e^{2 \gamma t}\left|u_{k}(t)\right|^{2} d t
\end{aligned}
$$

Using the Cauchy inequality we estimate the right-hand side of (4.28) as follows

$$
\int_{\sigma_{1}}^{\sigma_{2}} e^{2 \gamma t}\left(f_{k}(t), u_{k}(t)\right) d t \leq \varepsilon \int_{\sigma_{1}}^{\sigma_{2}} e^{2 \gamma t}\left|u_{k}(t)\right|^{2} d t+(4 \varepsilon)^{-1} \int_{\sigma_{1}}^{\sigma_{2}} e^{2 \gamma t}\left|f_{k}(t)\right|^{2} d t
$$


where $\varepsilon>0$ is arbitrary.

From (4.28), taking into account (4.30), (4.31) and (4.32), we obtain

$$
\begin{aligned}
\left.e^{2 \gamma t}\left|u_{k}(t)\right|^{2}\right|_{\sigma_{1}} ^{\sigma_{2}}+ & 2\left[\delta K_{1}-L-\gamma-\varepsilon\right] \int_{\sigma_{1}}^{\sigma_{2}} e^{2 \gamma t}\left|u_{k}(t)\right|^{2} d t \\
& +(1-\delta) K_{2} \int_{\sigma_{1}}^{\sigma_{2}} e^{2 \gamma t}\left\|u_{k}(t)\right\|^{2} d t \\
& +(1-\delta) \int_{\sigma_{1}}^{\sigma_{2}} e^{2 \gamma t} \Phi\left(u_{k}(t)\right) d t \\
\leq & (2 \varepsilon)^{-1} \int_{\sigma_{1}}^{\sigma_{2}} e^{2 \gamma t}\left|f_{k}(t)\right|^{2} d t, \quad \delta \in(0,1), \varepsilon \in(0,+\infty) .
\end{aligned}
$$

Since $K_{1}>0, \gamma$ satisfies (3.6), we first choose $\delta$ from $(0,1)$ such that $\delta K_{1}-L-\gamma>$ 0 , and then we choose $\varepsilon=2^{-1}\left[\delta K_{1}-L-\gamma\right]>0$. As a result, from (4.33) we obtain the estimate

$$
\begin{aligned}
\left.e^{2 \gamma t}\left|u_{k}(t)\right|^{2}\right|_{\sigma_{1}} ^{\sigma_{2}} & +\int_{\sigma_{1}}^{\sigma_{2}} e^{2 \gamma t}\left[|u(t)|^{2}+\left\|u_{k}(t)\right\|^{2}\right] d t+\int_{\sigma_{1}}^{\sigma_{2}} e^{2 \gamma t} \Phi\left(u_{k}(t)\right) d t \\
& \leq C_{3} \int_{\sigma_{1}}^{\sigma_{2}} e^{2 \gamma t}\left|f_{k}(t)\right|^{2} d t
\end{aligned}
$$

where $C_{3}$ is a positive constant depending on $K_{1}, K_{2}, L$ and $\gamma$ only.

We take $\sigma_{2}=\sigma \in S$ is arbitrary, and pass to the limit in (4.34) as $\sigma_{1} \rightarrow-\infty$. Taking into account $(\mathcal{F})$ and the definition of $u_{k}$ and $f_{k}$, we obtain

$$
\begin{aligned}
e^{2 \gamma \sigma}\left|u_{k}(\sigma)\right|^{2} & +\int_{-\infty}^{\sigma} e^{2 \gamma t}\left[|u(t)|^{2}+\left\|u_{k}(t)\right\|^{2}\right] d t \\
& +\int_{-\infty}^{\sigma} e^{2 \gamma t} \Phi\left(u_{k}(t)\right) d t \leq C_{3} \int_{-\infty}^{\sigma} e^{2 \gamma t}\left|f_{k}(t)\right|^{2} d t, \quad \sigma \in S .
\end{aligned}
$$

Since $\sigma \in S$ is arbitrary, from (4.35) it follows that

the sequence $\left\{u_{k}(\cdot)\right\}_{k=1}^{+\infty}$ is bounded in $L_{\gamma}^{\infty}(S ; H), L_{\gamma}^{2}(S ; H)$ and $L_{\gamma}^{2}(S ; V)$,

the sequence $\left\{e^{2 \gamma \cdot} \Phi\left(u_{k}(\cdot)\right)\right\}_{k=1}^{+\infty}$ is bounded in $L^{1}(S)$.

Now let us find estimates of $u_{k}^{\prime}, k \in \mathbb{N}$. For arbitrary fixed $k \in \mathbb{N}$ and almost every $t \in S$ we multiply equality (4.27) by $e^{2 \gamma t} u_{k}^{\prime}(t)$ and integrate the resulting equality from $\sigma_{1}$ to $\sigma_{2}$, where $\sigma_{1}, \sigma_{2} \in S$ are arbitrary numbers, $\sigma_{1}<\sigma_{2}$. From this we obtain

$$
\begin{aligned}
& \int_{\sigma_{1}}^{\sigma_{2}} e^{2 \gamma t}\left|u_{k}^{\prime}(t)\right|^{2} d t+\int_{\sigma_{1}}^{\sigma_{2}} e^{2 \gamma t}\left(g_{k}(t), u_{k}^{\prime}(t)\right) d t \\
& =\int_{\sigma_{1}}^{\sigma_{2}} e^{2 \gamma t}\left(f_{k}(t), u_{k}^{\prime}(t)\right) d t-\int_{\sigma_{1}}^{\sigma_{2}} e^{2 \gamma t}\left(B\left(t, u_{k}(t)\right), u_{k}^{\prime}(t)\right) d t
\end{aligned}
$$


Since $g_{k} \in L^{2}\left(\sigma_{1}, \sigma_{2} ; H\right)$, Lemma 4.1 implies that the function $\Phi_{H}\left(u_{k}(\cdot)\right)$ is absolutely continuous on $\left[\sigma_{1}, \sigma_{2}\right]$ and

$$
\frac{d}{d t} \Phi_{H}\left(u_{k}(t)\right)=\left(g_{k}(t), u_{k}^{\prime}(t)\right) \text { for a.e. } t \in\left(\sigma_{1}, \sigma_{2}\right) .
$$

Taking into account (4.39), we can rewrite the second term on the left side of (2.3) as follows

$$
\begin{aligned}
\int_{\sigma_{1}}^{\sigma_{2}} e^{2 \gamma t}\left(g_{k}(t), u_{k}^{\prime}(t)\right) d t & =\int_{\sigma_{1}}^{\sigma_{2}} e^{2 \gamma t} \frac{d}{d t} \Phi_{H}\left(u_{k}(t)\right) d t \\
& =\left.e^{2 \gamma t} \Phi_{H}\left(u_{k}(t)\right)\right|_{\sigma_{1}} ^{\sigma_{2}}-2 \gamma \int_{\sigma_{1}}^{\sigma_{2}} e^{2 \gamma t} \Phi_{H}\left(u_{k}(t)\right) d t .
\end{aligned}
$$

By the Cauchy inequality and (3.2) we have

$$
\begin{aligned}
\left|\int_{\sigma_{1}}^{\sigma_{2}} e^{2 \gamma t}\left(f_{k}(t), u_{k}^{\prime}(t)\right) d t\right| & \leq \int_{\sigma_{1}}^{\sigma_{2}} e^{2 \gamma t}\left|f_{k}(t)\right|\left|u_{k}^{\prime}(t)\right| d t \\
\leq & \frac{1}{4} \int_{\sigma_{1}}^{\sigma_{2}} e^{2 \gamma t}\left|u_{k}^{\prime}(t)\right|^{2} d t+\int_{\sigma_{1}}^{\sigma_{2}} e^{2 \gamma t}\left|f_{k}(t)\right|^{2} d t \\
\left|\int_{\sigma_{1}}^{\sigma_{2}} e^{2 \gamma t}\left(B\left(t, u_{k}(t)\right), u_{k}^{\prime}(t)\right) d t\right| & \leq \int_{\sigma_{1}}^{\sigma_{2}} e^{2 \gamma t}\left|B\left(t, u_{k}(t)\right)\right|\left|u_{k}^{\prime}(t)\right| d t \\
& \leq L \int_{\sigma_{1}}^{\sigma_{2}} e^{2 \gamma t}\left|u_{k}(t)\right|\left|u_{k}^{\prime}(t)\right| d t \\
& \leq L^{2} \int_{\sigma_{1}}^{\sigma_{2}} e^{2 \gamma t}\left|u_{k}(t)\right|^{2} d t+\frac{1}{4} \int_{\sigma_{1}}^{\sigma_{2}} e^{2 \gamma t}\left|u_{k}^{\prime}(t)\right|^{2} d t
\end{aligned}
$$

From (2.3), taking into account (4.40), (4.41), (4.42), we obtain

$$
\begin{aligned}
\frac{1}{2} \int_{\sigma_{1}}^{\sigma_{2}} e^{2 \gamma t}\left|u_{k}^{\prime}(t)\right|^{2} d t+\left.e^{2 \gamma t} \Phi_{H}\left(u_{k}(t)\right)\right|_{\sigma_{1}} ^{\sigma_{2}} \\
\quad \leq L^{2} \int_{\sigma_{1}}^{\sigma_{2}} e^{2 \gamma t}\left|u_{k}(t)\right|^{2} d t \\
\quad+2 \gamma \int_{\sigma_{1}}^{\sigma_{2}} e^{2 \gamma t} \Phi_{H}\left(u_{k}(t)\right) d t+\int_{\sigma_{1}}^{\sigma_{2}} e^{2 \gamma t}\left|f_{k}(t)\right|^{2} d t
\end{aligned}
$$

By the definitions of $u_{k}$ and $f_{k}$ we pass to the limit in (4.43) when $\sigma_{1} \rightarrow-\infty$. From obtained inequality, taking into account estimate (4.35), setting $\sigma_{2}=\sigma \in S$, we have

$$
e^{2 \gamma \sigma} \Phi_{H}\left(u_{k}(\sigma)\right)+\int_{-\infty}^{\sigma} e^{2 \gamma t}\left|u_{k}^{\prime}(t)\right|^{2} d t \leq C_{4} \int_{-\infty}^{\sigma} e^{2 \gamma t}\left|f_{k}(t)\right|^{2} d t
$$

where $C_{4}$ is a positive constant depending on $K_{1}, K_{2}, L$ and $\gamma$ only. 
According to the definitions of the functional $\Phi_{H}$ and the function $f_{k}$, and condition $\left(\mathcal{A}_{4}\right)$ (recall that $u_{k}(t) \in V$ for a.e. $t \in S$ ), from (4.44) we obtain

$$
e^{2 \gamma \sigma}\left\|u_{k}(\sigma)\right\|^{2}+\int_{-\infty}^{\sigma} e^{2 \gamma t}\left|u_{k}^{\prime}(t)\right|^{2} d t \leq C_{5} \int_{-\infty}^{\sigma} e^{2 \gamma t}|f(t)|^{2} d t
$$

where $C_{5}>0$ is a constant depending on $K_{1}, K_{2}, L$, and $\gamma$ only.

Estimate (4.45) imply that

the sequence $\left\{u_{k}\right\}_{k=1}^{+\infty}$ is bounded in $L_{\gamma}^{\infty}(S ; V)$,

the sequence $\left\{u_{k}^{\prime}\right\}_{k=1}^{+\infty}$ is bounded in $L_{\gamma}^{2}(S ; H)$.

Let us show that

$$
\text { the sequence }\left\{g_{k}\right\}_{k=1}^{+\infty} \text { is bounded in } L_{\gamma}^{2}(S ; H) \text {. }
$$

Indeed, using (3.2) and (4.35) we have

$$
\int_{\sigma_{1}}^{\sigma_{2}} e^{2 \gamma t}\left|B\left(t, u_{k}(t)\right)\right|^{2} d t \leq L^{2} \int_{\sigma_{1}}^{\sigma_{2}} e^{2 \gamma t}\left|u_{k}(t)\right|^{2} d t \leq C_{6},
$$

where $C_{6}>0$ is a constant independing on $k \in \mathbb{N}, \sigma_{1}, \sigma_{2} \in S$.

Therefore, from $(4.27),(4.47),(4.49),(\mathcal{F})$ and the definition of $f_{k}$ we obtain (4.48)

Step 4 (passing to the limit). Since $V$ and $H$ are Hilbert spaces, and $V$ embeds in $H$ by compact injection, from (4.36), (4.46), (4.47), (4.48) and Lemma 2.4 we have that there exist functions

$$
u \in L_{\gamma}^{\infty}(S ; V) \cap L_{\gamma}^{2}(S ; V) \cap H_{\gamma}^{1}(S ; H), \quad g \in L_{\gamma}^{2}(S ; H)
$$

and a subsequence of the sequence $\left\{u_{k}, g_{k}\right\}_{k=1}^{+\infty}$ (still denoted by $\left.\left\{u_{k}, g_{k}\right\}_{k=1}^{+\infty}\right)$ such that

$$
\begin{aligned}
& e^{\gamma \cdot} u_{k}(\cdot) \underset{k \rightarrow \infty}{\longrightarrow} e^{\gamma \cdot} u(\cdot) \quad \text { *-weakly in } L^{\infty}(S ; V), \\
& u_{k} \underset{k \rightarrow \infty}{\longrightarrow} \quad \text { weakly in } L_{\gamma}^{2}(S ; V) \text { and weakly in } H_{\gamma}^{1}(S ; H), \\
& u_{k} \underset{k \rightarrow \infty}{\longrightarrow} \quad \text { in } C(S ; H), \\
& g_{k} \underset{k \rightarrow \infty}{\longrightarrow} g \quad \text { weakly in } L_{\gamma}^{2}(S ; H) .
\end{aligned}
$$

Note that (4.51) and (4.53) imply

$$
u_{k} \underset{k \rightarrow \infty}{\longrightarrow} u, \quad u_{k}^{\prime} \underset{k \rightarrow \infty}{\longrightarrow} u^{\prime}, \quad g_{k} \underset{k \rightarrow \infty}{\longrightarrow} g \text { weakly in } L_{\text {loc }}^{2}(S ; H) .
$$

Using (3.1) and (4.52), for each $\sigma<0$ we obtain

$$
\int_{\sigma}^{0}\left|B\left(t, u_{k}(t)\right)-B(t, u(t))\right|^{2} d t \leq L^{2} \int_{\sigma}^{0}\left|u_{k}(t)-u(t)\right|^{2} d t \underset{k \rightarrow \infty}{\longrightarrow} 0 .
$$


Thus, we obtain

$$
B\left(\cdot, u_{k}(\cdot)\right) \underset{k \rightarrow \infty}{\longrightarrow} B(\cdot, u(\cdot)) \text { strongly in } L_{\text {loc }}^{2}(S ; H) .
$$

Let $v \in H, \varphi \in D(-\infty, 0)$ be arbitrary. For a.e. $t \in S$ we multiply equality (4.27) by $v$, and then we multiply the obtained equality by $\varphi$ and integrate in $t$ on $S$. As a result, we obtain the equality

$$
\begin{aligned}
\int_{S}\left(u_{k}^{\prime}(t), v \varphi(t)\right) d t & +\int_{S}\left(g_{k}(t), v \varphi(t)\right) d t+\int_{S}\left(B\left(t, u_{k}(t)\right), v \varphi(t)\right) d t \\
& =\int_{S}\left(f_{k}(t), v \varphi(t)\right) d t, \quad k \in \mathbb{N} .
\end{aligned}
$$

We pass to the limit in (4.57) as $k \rightarrow \infty$, taking into account (4.54), (4.56) and convergence of $\left\{f_{k}\right\}$ to $f$ in $L_{\mathrm{loc}}^{2}(S ; H)$. As a result, since $v \in H, \varphi \in D(-\infty, 0)$ are arbitrary, for a.e. $t \in S$ we obtain the equality

$$
u^{\prime}(t)+g(t)+B(t, u(t))=f(t) \quad \text { in } H
$$

Step 5 (completion of proof). In order to complete the proof of the theorem it remains only to show that $u(t) \in D(\partial \Phi)$ and $g(t) \in \partial \Phi(u(t))$ for a.e. $t \in S$.

Let $k \in \mathbb{N}$ be an arbitrary number. Since $u_{k}(t) \in D\left(\partial \Phi_{H}\right)$ and $g_{k}(t) \in$ $\partial \Phi_{H}\left(u_{k}(t)\right)$ for every $t \in S \backslash \widetilde{S}_{k}$, where $\widetilde{S}_{k} \subset S$ is a set of measure zero, applying the monotonicity of the subdifferential $\partial \Phi_{H}$, we obtain that for every $t \in S \backslash \widetilde{S}_{k}$ the following equality holds

$$
\left(g_{k}(t)-v^{*}, u_{k}(t)-v\right) \geq 0, \quad \forall\left[v, v^{*}\right] \in \partial \Phi_{H} .
$$

Let $\sigma \in S, h>0$ be arbitrary numbers. We integrate (4.58) on $(\sigma-h ; \sigma)$ :

$$
\int_{\sigma-h}^{\sigma}\left(g_{k}(t)-v^{*}, u_{k}(t)-v\right) d t \geq 0, \quad \forall\left[v, v^{*}\right] \in \partial \Phi_{H} .
$$

Now according to (4.52) and (4.53) we pass to the limit in (4.59) as $k \rightarrow \infty$. As a result we obtain

$$
\int_{\sigma-h}^{\sigma}\left(g(t)-v^{*}, u(t)-v\right) d t \geq 0, \quad \forall\left[v, v^{*}\right] \in \partial \Phi_{H} .
$$

The monograph [27, Theorem 2, p. 192] and (4.60) imply that for every $\left[v, v^{*}\right] \in \partial \Phi_{H}$ there exists a set $R_{\left[v, v_{*}\right]} \subset S$ of measure zero such that for all $\sigma \in S \backslash R_{\left[v, v_{*}\right]}$ we have

$$
0 \leq \lim _{h \rightarrow+0} \frac{1}{h} \int_{\sigma-h}^{\sigma}\left(g(t)-v^{*}, u(t)-v\right) d t=\left(g(\sigma)-v^{*}, u(\sigma)-v\right) .
$$

Let us show that there exists a set of measure zero $R \subset S$ such that

$$
\forall \sigma \in S \backslash R: \quad\left(g(\sigma)-v^{*}, u(\sigma)-v\right) \geq 0, \quad \forall\left[v, v^{*}\right] \in \partial \Phi_{H} .
$$


Since $V$ and $H$ are separable spaces, there exists a countable set $F \subset \partial \Phi_{H} \subset$ $V \times H$ which is dense in $\partial \Phi_{H}$. Let us denote $R:=\underset{\left[v, v^{*}\right] \in F}{\cup} R_{\left[v, v_{*}\right]}$. Since the set $F$ is countable, and any countable union of sets of measure zero is a set of measure zero, $R$ is a set of measure zero. Therefore, for any $\sigma \in S \backslash R$ inequality $\left(g(\sigma)-v^{*}, u(\sigma)-v\right) \geq 0$ holds for every $\left[v, v^{*}\right] \in F$. Let $\left[\widehat{v}, \widehat{v}^{*}\right]$ be an arbitrary element from $\partial \Phi_{H}$. Then from the density $F$ in $\partial \Phi_{H}$ we have the existence of a sequence $\left\{\left[v_{l}, v_{l}^{*}\right]\right\}_{l=1}^{\infty}$ such that $v_{l} \rightarrow \widehat{v}$ in $V, v_{l}^{*} \rightarrow \widehat{v}^{*}$ in $H$ and

$$
\forall \sigma \in S \backslash R: \quad\left(g(\sigma)-v_{l}^{*}, u(\sigma)-v_{l}\right) \geq 0 \quad \forall l \in \mathbb{N} .
$$

Thus, passing to the limit in this equality as $l \rightarrow \infty$, we get $\left(g(\sigma)-\widehat{v}^{*}, u(\sigma)-\widehat{v}\right) \geq 0$ $\forall \sigma \in S \backslash R$. Therefore, inequality (4.62) holds. From this, according to maximal monotonicity of $\partial \Phi_{H}$, we obtain that $[u(t), g(t)] \in \partial \Phi_{H}$ for a.e. $t \in S$.

Estimate (3.7) of the solution of the problem $\mathbf{P}(\Phi, B, f, \gamma)$ follows directly from (4.35), (4.45), (4.50), (4.51) and (4.52), Lemma 2.2, Fatou's Lemma and the fact that $\Phi_{H}$ is lower semicontinuous in $H$.

From (4.35) we have

$$
e^{2 \gamma \sigma}|u(\sigma)|^{2} \leq C_{3} \int_{-\infty}^{\sigma} e^{2 \gamma t}|f(t)|^{2} d t
$$

This inequality and condition $(\mathcal{F})$ imply that $u$ satisfies condition (3.4). Thus Theorem 3.2 is proved.

\section{References}

1. J.-P. Aubin, Un theoreme de compacite, Comptes rendus hebdomadaires des seances de l'academie des sciences, 256, (24) (1963), 5042-5044.

2. F. BERNIS, Existence results for doubly nonlinear higher order parabolic equations on unbounded domains, Mathematische Annalen, 279 (1988), 373-394.

3. M. Bokalo, Problem without initial conditions for some classes of nonlinear parabolic equations, Journal of Soviet Mathematics, 51 (1990), 2291-2322.

4. M. BoKalo, Well-posedness of problems without initial conditions for nonlinear parabolic variational inequalities, Nonlinear Boundary Problem, 8 (1998), 58-63.

5. M. Bokalo, V. Dmytriv, Boundary problems for integro-differential equations in anisotropic spaces, Visnyk of the Lviv Univ. Series Mech. Math., 59 (2001), 84-101 (in Ukrainian).

6. M. Bokalo, Yu. Dmytryshyn, Problems without initial conditions for degenerate implicit evolution equations, Electronic Journal of Differential Equations, 2008, (4) (2008), 1-16.

7. M. BokAlo, A. LoREnzI, Linear evolution first-order problems without initial conditions, Milan Journal of Mathematics, 77 (2009), 437-494.

8. M. BoKalo, Almost periodic solutions of anisotropic elliptic-parabolic equations with variable exponents of nonlinearity, Electronic Journal of Differential Equations, 2014 (178) (2014), 1-13.

9. M. Bokalo, I. SKIRA, Almost periodic solutions for nonlinear integro-differential elliptic-parabolic equations with variable exponents of nonlinearity, International Journal of Evolution Equations, 10 (3-4) (2017), 297-314. 
10. M. BokAlo, I. SKIRA, Well-posedness of the Fourier problem for higher-order weakly nonlinear integro-differential elliptic-parabolic equations, Visnyk of the Lviv Univ. Series Mech. Math., 85 (2018), 91-106 (in Ukrainian).

11. H. BRÉZIS, Opérateurs maximaux monotones et semi-groupes de contractions dans les espaces de Hilbert, North-Holland Publishing Comp., Amsterdam, London, 1973.

12. H. Brezis, Functional Analysis, Sobolev Spaces and Partial Differential Equations, Springer, New York, Dordrecht, Heidelberg, London, 2011.

13. O. BuHRII, Some parabolic variational inequalities without initial conditions, Visnyk of the Lviv University. Series Mechanics and Mathematics, 49 (1998), 113-121.

14. H. Gajewski, K. GrÖGer, K. ZaCharias, Nichtlineare Operatorgleichungen und Operatordifferentialgleichungen, Akademie-Verlag, Berlin, 1974.

15. S. D. Ivasishen, Parabolic boundary-value problems without initial conditions, Ukrainian Mathematical Journal, 34 (5) (1982), 547-552.

16. J.-L. Lions, Quelques méthodes de résolution des problémes aux limites non linéaires, Dunod Gauthier-Villars, Paris (France), 1969.

17. O. OleiniK, G. IosifJan, Analog of Saint-Venant's principle and uniqueness of solutions of the boundary problems in unbounded domain for parabolic equations, Uspekhi Mat. Nauk, 31 (6) (1976), 142-166.

18. A. PANKOv, Bounded and almost periodic solutions of nonlinear operator differential equations, Kluwer, Dordrecht, 1990.

19. P.YA. PUKACH, On problem without initial conditions for some nonlinear degenarated parabolic system, Ukrainian Mathematical Journal, 46 (4) (1994), 484-487.

20. R. RockAfEllar, On the maximal monotonicity of subdifferential mappings, Pacific Journal of Mathematics, 33 (1) (1970), 209-216.

21. R. E. Showalter, Singular nonlinear evolution equations, The Rocky Mountain Journal of Mathematics, 10 (3) (1980), 499-507.

22. R. E. SHOWALTER, Monotone operators in Banach space and nonlinear partial differential equations, American Mathematical Society, 49, Providence, 1997.

23. A. Tychonoff, Théorèmes d'unicité pour l'équation de la chaleur, Mat. Sb., 42 (2) (1935), 199-216.

24. O. BuHRII, N. BuHRII, On initial-boundary value problem for nonlinear integrodifferential equations with variable exponents of nonlinearity, New Trends in Mathematical Sciences, 5 (3) (2017), 128-153.

25. O. BuHRII, N. BuhrII, Integro-differential systems with variable exponents of nonlinearity, Open Mathematics, 15 (2017), 859--883.

26. M. LOAYZA, Asymptotic behavior of solutions to parabolic problems with nonlinear nonlocal terms, Electronic Journal of Differential Equations, 2013 (228) (2013), 1-12.

27. K. YoshidA, Functional Analysis, Springer-Verlag, Berlin Heidelberg, 1995. 